\title{
Phytochemicals Extraction and Nutraceuticals of Purple Corn
}

\author{
W. Puminat ${ }^{1 *} \&$ C. Teangpook ${ }^{2}$ \\ ${ }^{1}$ Division of Food Chemistry and Physic, Institution of Food Research and Product Development, \\ Kasetsart University, Kasetsart, Bangkok, Thailand \\ ${ }^{2}$ Division of Food Processing and Preservation, Institution of Food Research and Product Development, \\ Kasetsart University, Kasetsart, Bangkok, Thailand \\ *W. Puminat, E-mail: ifrwnp@ hotmail.com
}

Received: October 19, 2017 Accepted: October 25, 2017 Online Published: November 3, 2017 doi:10.22158/fsns.v1n2p104

URL: http://dx.doi.org/10.22158/fsns.v1n2p104

\begin{abstract}
Phytochemicals are naturally formed in plant of corn. Many kinds of flavonoids are the most important plant pigment. Purple corn is extracted with polar and nonpolar solvent and analyzed for flavonoids and antioxidants. The extract residue of purple corn is compared on extraction with various solvent groups. In the designed experiments, conditions of extraction depend on solvent, solvent ratio and evaporation temperature. The residue quantity of purple corn is respectively from extraction with three kinds of solvent such as acetone, chloroform: methanol (3:1) and pentane: hexane (1:1). All residue extract are eluted and with mobile phase and analyzed with diode array detector by HPLC. Anthocyanin, $\beta$-carotene and total tannin of purple corn were taken analysis an average (mean $\pm S D$ ) in $100 \mathrm{~g}$ sample and shown as $198.42 \pm 0.33 \mathrm{mg}, 175.82 \pm 0.17 \mathrm{mg}$ and $273.75 \pm 0.33 \mathrm{mg}$ respectively. The efficiency of solvent extraction depends on the polarity of the substances. The extract by acetone can take the highest residue and mixture solvent take a high residue. The different polarities of solvent can make the difference of residue product in the extraction. Improving and development on the changes are useful for the best of raw material and products.
\end{abstract}

\section{Keywords}

purple corn, nutraceuticals, extraction and anthocyanin

\section{Introduction}

Flavonoids are naturally formed in plant. Flavonoids are widely distributed in plants, fulfilling many functions. Some flavonoids are phenolic substances that act in plant as antioxidants. Many kinds of flavonoids are the most important plant pigments. Plant pigments include a variety of different kinds of molecule, including porphyrins, carotenoids, anthocyanins and betalains. Purple pigments of purple corn have the properties of free radicals and reduce symptoms of cancer tumor types. They enhance the strengthen of body, increase an immunity antibodies and the red blood cells. They reduce the fat in 
artery clogs, prevent heart disease and control blood sugar levels. Pigments color help anti-aging, the digestive system and deterioration of eye.

Physical and chemical changes with growth, this is useful in helping to identify compounds in pigments. If we know the chemical properties of substances, we are likely to predict what kind of reaction. We see a chemical change based on certain chemical properties of pigments. These pigments are generally measured by determining the amount and wavelength of light that they absorb. The wavelength and amount of absorbed light can show the identify and content of pigments. All substances have properties that functional and identifying. we can utilize by separating them from one another by extraction with solvents and purity by chromatography.

Purple corn has a rich composition of phytochemicals such as anthocyanins and phenolic compounds. The phytonutrients are natural compounds or substances found in certain plants which is believed to be beneficial to human health and help prevent various diseases. This class of phytonutrients includes pigments such as carotenoids (beta-carotene, lutein), flavonoids or phenolics, alkaloids, nitrogen-containing compounds and organosulfur compounds (indoles, glucosinolates). The color categories of phytonutrients are red, red purple (and blue), orange, orange-yellow, yellow-green, green and white-green.

Liquid extraction also known as solvent extraction is a method to separate compounds based on their relative solubilities by preferentially dissolving that substance in a suitable solvent. Generally, polar solvents dissolve polar compounds and non-polar solvents dissolve non-polar compounds. Strongly polar compounds dissolve only in very polar, while strongly non-polar compounds dissolve only in very non-polar organic solvents. Polar and non-polar are not miscible with each other and will quickly separate into two layers even after being shaken well. The type of polarity, dipole moment, and hydrogen bonding of solvent is able to specify the dissolve.

\section{Materials and Methods}

\subsection{The Characteristics and Physical Properties of Purple Corn}

This classification scheme will be based on their shapes and structures for morphological classification. Physical characteristics are defining traits or features about general quality. The samples of purple corn are classified and weighted for determining an average of groups. A classification is provided the groups in order to understand the general standard of purple corn.

\subsubsection{Preparation of Purple Corn Powder and Drying}

Fresh purple corn is sliced in size 1-2 mm and crushed to a fine shred. Sample is taken drying by vacuum drying at $60^{\circ} \mathrm{C}$ for $7 \mathrm{hr}$. The samples are pulverized to a fine powder and stored in seal container for further analysis and the other process.

\subsubsection{Determination of Moisture Content}

Fresh purple corn is sliced in size 1-2 mm and dry with the vacuum oven. Moisture is determined by drying 1-3 g sample in a vacuum oven $(25-100 \mathrm{mmHg})$ at $60^{\circ} \mathrm{C}$ for $7 \mathrm{hr}$. The moisture content of 
sample is calculated as percentage of the different weight.

\subsubsection{Measurement the Colour of Purple Corn}

Sample is measured in three times and seven sampling. Colour measuring is taken with the Data Colour International Measurement model Colour Tools. CIE value (Commission Internationale de I'Eclaerage) display the colour value in CIELAB system $L^{*}(0=$ black and $100=$ white $), a^{*}\left(-a^{*}=\right.$ green and $+a^{*}=$ red) and $b^{*}\left(-b^{*}=\right.$ blue and $+b^{*}=$ yellow) at D65 10Deg (Light source Illuminant D).

\subsection{Analysis of Nutraceuticals Purple Corn}

\subsubsection{Determination Total Anthocyanin by pH-Differential Method}

Total anthocyanin content is extracted and determined by UV-VIS spectroscopy. The extract is added to test tubes containing of buffers $\mathrm{pH} 1.0$ and $\mathrm{pH}$ 4.5. They are thoroughly mixed by vortex mixer. Transperent sample mixer is measured at different $\mathrm{pH}$ with an absorbance at wavelength $530 \mathrm{~nm}$ and $700 \mathrm{~nm}$ by UV-Visible spectrophotometer. Total anthocyanin content is calculated and determined as anthocyanin (cyanidin) in milligram/100 gram by the following equation $\%$ anthocyanin pigments $(\mathrm{mg} / \mathrm{l})=\mathrm{A} * \mathrm{MW} * \mathrm{DF} * 1000 / \varepsilon * 1 ; \mathrm{A}=(\mathrm{A} 530 \mathrm{~nm}-\mathrm{A} 700 \mathrm{~nm}) \mathrm{pH} 1-(\mathrm{A} 530 \mathrm{~nm}-\mathrm{A} 700 \mathrm{~nm}) \mathrm{pH} 4.5$. A is a difference of absorbance at $\mathrm{pH}$ 1and $\mathrm{pH}$ 4.5. MW. is molecular weight (cyanidin-3-glucoside $=449.2$ $\mathrm{g} / \mathrm{mol})$ and $\varepsilon$ is the molar absorptivity for cyanidin-3-glucoside $(\varepsilon=26900 \mathrm{~L} / \mathrm{mol} / \mathrm{cm}) .1$ is standard of pathlength $1 \mathrm{~cm}$ and $\mathrm{DF}$ is dilution factor.

\subsubsection{Determination Pigment Content and Pigments Extract}

Each sample is prepared by $5 \mathrm{~g}$ pasted purple corn immersed in 1-butanol $25 \mathrm{~mL}$. They are extracted in an ultrasonic water bath for $2 \mathrm{hrs}$, and centrifuged at 4,500 rpm for $20 \mathrm{~min}$. The upper layer is supernatant for pigment analysis by uv-visible spectrophotometer. The absorbance at $435 \mathrm{~nm}$ show pigment content of purple corn.

\subsubsection{Determination $\beta$-Carotene Extract by uv-visible Spectrophotometer}

Purple corn are extracted with hexane and acetone. Sample $1 \mathrm{~g}$ is added with $15 \mathrm{ml}$ hexane: acetone (2:3). Mixture is soaked and shaken with vortex mixer for 10 minutes. They are allowed to stand by magnesia adsorber and centrifuged at $4500 \mathrm{rpm}$ for 10 minutes. The extract is separated in vial with cap and measured by spectrophotometry at wavelength $436 \mathrm{~nm}$. The determination of $\beta$-carotene is compared and calculated with standard solution. The standard curve of $\beta$-carotene is prepared at concentrations of $0.001-0.015 \mathrm{mg} / \mathrm{ml}$. The concentrations are calculated using a linear regression of the five points in standard curve.

\subsubsection{Determination of Polyphenol or Total Phenolic Assay}

Total phenolic compounds are quantified as tannin content by using Folin Ciocalteu's method. Sample is extracted with water and filtered by filter paper. Extract is formed a complex compound with Folin-Ciocalteu reagent. Polyphenol or tannin is analyzed by spectrophotometer. Blue colour compound is determined an absorbance at wavelength $760 \mathrm{~nm}$. Blank is prepared by replacing the reagent on the same way by sample and standard reference. Samples are compared with standard calibration of tannin and calculated as total tannin content. 


\subsubsection{Determination of Amylose Content in Purple Corn}

The samples are dissolved with urea and dimethylsulfoxide (UDMSO) in a water bath at high temperature until changed in gel formulation. Contaminated fat is eluted with alcohol and dried in oven. Residues without ethanol are dissolved UDMSO again and adjusted the volume with water. Solution is formed the color with iodine in potassium iodide solution and measured the absorbance at $635 \mathrm{~nm}$ wavelength. Absorbance is compared with standard reference and calculated for amylose content.

\subsection{Extraction and Solvent Extract}

In the designed experiments on extraction model, conditions of extraction for the yield depend on solvent, solvent ratio and evaporation temperature. Control on the extract ratio is 1:7 wt/ml. Sample is approximately weighted in $10 \mathrm{~g}$ and mixed with $70 \mathrm{ml}$ solvent extract. It is tightly closed the lid and shaken a mixture. Controls on the condition are the same temperature and shaker at speed $180 \mathrm{rpm}$ for 8 hour. The suspension is filtered through filter paper. All of extract liquids are done the evaporation of solvent by Buchi Syncorne Analyst in three stage of temperature at $40^{\circ} \mathrm{C}, 50^{\circ} \mathrm{C}$ and $60^{\circ} \mathrm{C}$. The evaporation is controlled by temperature, vacuum pressure and speed controller at $150 \mathrm{rpm}$ until dryness. Residue is blowed with nitrogen gas in order to evaporate the remained solvent. The specific substances are focused on weight of recovery residue.

$\%$ Extracted $=$ Total residue $* 10 /$ wt.sample

The extract in each experiment can be compared the differences and taken for the other analysis.

\subsection{Identification of Flavonoids and Antioxidants by HPLC}

\subsubsection{Sample and Preparation Sample}

Analysis of anthocyanin is determined by HPLC (High Performance Liquid Chromatography). Residue is weighted and mixed with $25 \mathrm{ml}$ ethanol. It is tightly closed the lid and shaken to dissolve by a vortex mixer. The sample is extracted with ultrasonic agitation for $15 \mathrm{~min}$ and shaken with a vortex mixer again. Solution is precipitated with centrifuge (Minicentrifuge C 1200). The upper layer of solution is followed by filter membrane $\phi 0.45 \mu \mathrm{m}$ and injected for $1 \mathrm{microL}$ into the HPLC.

\subsubsection{Determining of Flavonoids and Total Flavonoids}

Conditions of analysis in grouping, mobile phase is acetonitrile: 5\% formic acid: methanol: water; 75 : 10: 10: 5 and elute with column at flow rate $0.9 \mathrm{ml} / \mathrm{min}$. Column is ODS Hypersil particle size $5 \mu \mathrm{m} \mathrm{ID}$ $4.6 \mathrm{~mm}$ length $250 \mathrm{~mm}$ and detect the peak with diode array detector at a wavelength $280 \mathrm{~nm}$. The concentration of cyanidin-3-glucoside standard reference is level 1.5-6 ng for determining as flavonoids and another series.

\subsubsection{Determining of Antioxidants and Total Antioxidants}

Antioxidant analysis, extracted sample is carried out by HPLC analysis with UV detector. Mobile phase is acetonitrile: methanol; 90: 10 and elute column at flow rate $0.7 \mathrm{ml} / \mathrm{min}$. Both antioxidants and flavonoids analysis detected on the same wavelength and column. The analysis is determined at room temperature and identified the chromatogram in series of epicatechin. 


\subsection{Statistical Analysis}

The data is analysed by statistical program of ANOVA (Analysis of Variance). Statistical analysis of mean and variance in each treatment are taken with Duncan's new multiple range test at the significance 0.01 .

\section{Results and Discussions}

\subsection{Evaluation of Physical and Chemical Properties}

The physical and chemical characteristics are a defining of traits about qualification. Their identities are sometimes taken into consideration to evaluate certain corn. In the experiments, their properties are classified as either extensive or intensive. Extensive properties depend on the amount of matter in the sample such as colour, \% silk or pollen, average weight per three pods. Intensive properties do not depend on the sample size or mass such as pigment, anthocyanin, tannin and amylose. Mean and standard deviation (Mean \pm SD.) of $\%$ weight of purple corn are pod $46.16 \pm 0.57 \%$, silk or pollen $19.88 \pm 2.71 \%$, peel $33.96 \pm 2.55 \%$ and bulk weight per three pod $1.257 \pm 0.015 \mathrm{~kg}$.

\subsubsection{Moisture Content and Dry Sample}

An average (Mean \pm SD.) moisture of fresh corn and silk is about $73.40 \pm 1.01 \%$ and $24.47 \pm 0.40$ respectively. Drying sample is prepared by heating in vacuum oven. The moisture is removed by evaporation of water. Moisture in the sample is measured by the difference of mass before and after drying. The moisture content of the sample is calculated as percentage of the different weight loss. An average moisture of drying powder is about $12.19 \pm 0.02 \%$. The samples are pulverized to a fine powder and stored in seal container for further analysis and process.

\subsubsection{Purple Colour of Corn and Colour Value (CIELAB)}

The pollen or silk has a high red colour but seed has a high blue colour. Colour in the CIELAB system ( $\mathrm{L}^{*}, \mathrm{a}^{*}$ and $\mathrm{b}^{*}$ ) of purple corn in seed and silk are different a statistical significance at $\alpha=0.05$. The relationships between the association of two variables are shown with Pearson's correlation. Table 1 is shown Pearson's correlation of colour and some characteristics of purple corn. In each part of corn pod and colour value in the table are compared the average and a variance on random analysis by statistics at significant level $\alpha=0.05$.

Table 1. The Characteristics and Colour Value of Purple Corn and Its Silk

\begin{tabular}{|c|c|c|c|c|c|}
\hline \multirow[t]{2}{*}{ Purple corn } & \multirow{2}{*}{$\begin{array}{l}\text { Total weight } \\
\text { (kg) per } 3 \text { pod }\end{array}$} & \multicolumn{3}{|c|}{ Colour value } & \multirow[b]{2}{*}{$\mathbf{C}$} \\
\hline & & $\mathbf{L}^{*}$ & $\mathbf{a}^{*}$ & $\mathbf{b}^{*}$ & \\
\hline Kenel and seed & $1.257 \pm 0.017$ & $18.964 \pm 3.924^{\mathrm{a}}$ & $2.047 \pm 0.746^{\mathrm{a}, \mathrm{b}}$ & $0.742 \pm 0.480^{\mathrm{a}}$ & $2.282 \pm 0.523^{b}$ \\
\hline Silk & $0.250 \pm 0.038$ & $20.173 \pm 3.49^{c}$ & $3.647 \pm 2.052^{\mathrm{c}}$ & $0.520 \pm 0.585^{\mathrm{c}, \mathrm{d}}$ & $3.807 \pm 1.784^{\mathrm{d}}$ \\
\hline
\end{tabular}


In Table 1, a,c and b,d show the relationships according to Duncan's multiple range test at $\alpha=0.05$ and $\alpha=0.01$. Colour value (CIELAB) in each column are significantly different at $\alpha=0.05$.

\subsection{Evaluation on Nutraceuticals of Purple Corn}

Anthocyanins are a group of naturally occurring flavonoid compounds, which play an important role in the colour quality of purple corn. In the experiments, the analysis of extract has respectively anthocyanin and pigment an average (Mean \pm SD) $198.42 \pm 0.33 \mathrm{mg} / 100 \mathrm{~g}$ and $204.52 \pm 0.30 \mathrm{mg} / 100$ g. Tannin and $\beta$-carotene of purple corn are taken analysis and shown as fresh, frozen and drying sample in Table 2.

Table 2. Some Phytonutients of Fresh Corn, Frozen Corn and Dry Corn

\begin{tabular}{llll}
\hline Phytonutrients & Fresh Corn $($ Mean \pm SD) & Frozen Corn $($ Mean \pm SD) & Dry Corn $($ Mean \pm SD) \\
\hline Tannin $(\mathrm{mg} / 100 \mathrm{~g})$ & $273.75 \pm 0.33$ & $259.55 \pm 0.48$ & $1274.32 \pm 0.49$ \\
$\beta$-carotene $(\mathrm{mg} / 100 \mathrm{~g})$ & $175.82 \pm 0.17$ & $156.60 \pm 0.02$ & $496.54 \pm 0.19$ \\
\hline
\end{tabular}

Standard cruve of linear equation and $\mathrm{R}$-squared value (tannin and $\beta$-carotene) in puple corn are respectively displayed as $y=0.2244 x-0.0066: R^{2}=0.9958$ and $y=1.9256 x-0.0129: R^{2}=0.9899$. In the experiments, amylose of fresh purple corn is $121.75 \pm 0.32 \mathrm{mg} / 100 \mathrm{~g}$ and drying powder average $834.35 \pm 0.75 \mathrm{mg} / 100 \mathrm{~g}$. The calibration graph is shown a linearity, with a correlation coefficient of $\mathrm{R}^{2}$ $=0.9986$. The representative linear of regression equation for standard amylose is $\mathrm{y}=0.1966 \mathrm{x}+$ 0.0261 .

\subsection{Solvent Extraction and Residue of Purple Corn with Various Solvent Extract}

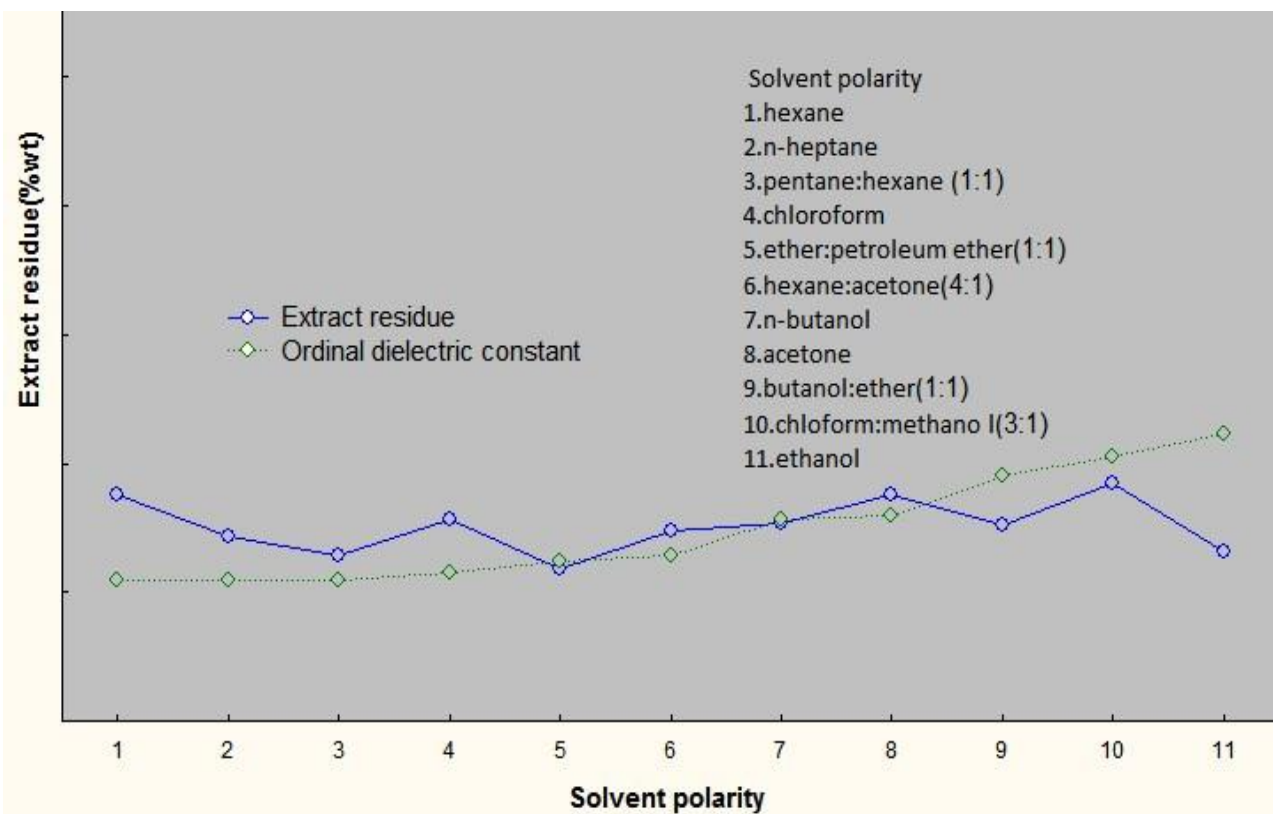

Figure 1. The Extract Residues of the Various Solvent Polarities 


\subsubsection{Effect of Solvent on the Extraction of Nutraceuticals in Purple Corn}

All substances have properties as functionals and nutraceuticals. The crude extract is identified and analyzed for determination of flavonoids and antioxidants. The polarity of the substances are compared to the different extraction. The residue content is evaluated by comparing with various solvent extract. By separating them by extraction with solvents, difference of nutraceuticals are a process that substances dissolve selectively by one or more of the mixture of solvent. In the experiments, mixture solvent of chloroform and methanol is the best described system for partially polar and nonpolar can extract to high residue. The relationships of residue and solvent polarity in extraction of purple corn show on Figure 1.

\subsubsection{Residue Extract and Solvent Polarities in the Extraction of Purple Corn}

Figure 1 shows the different types of weight $(\%)$ of chemical composition by priority of polar extraction. They are found that the extract residue have higher yield than another extract solvent. If the dielectric constant of the extract is divided into three groups the difference of extract residue is from the solvent polarity. It is found that they have statistically a difference at significant level $\alpha=0.05$.

Table 3. Capacity and Potential of Solvent Extract for the Extraction of Purple Corn

\begin{tabular}{lll}
\hline Solvent extract & Focus on Functional groups & \% Crude residue (g/ml) \\
\hline Acetone & Pigments & 10.682 \\
Chloform: Methanol; 3:1 & The best partially polar and nonpolar & 7.693 \\
Pentane +hexane; 1:1 & Fat \& Oil & 7.644 \\
Ether + Petroleum ether; 1:1 & Fat \& Oil & 5.635 \\
Butanol + Ether; 1:1 & Pigments & 5.635 \\
Hexane & Non polar & 5.586 \\
Ethanol & Sugar & 4.018 \\
Chloroform & Soluble matter & 2.205 \\
n-butanol & Colour & 0.763 \\
hexane + Acetone; 4:1 & Fat \& Oil & 0.847 \\
n-butanol & Colour & 0.763 \\
n-heptane & Unsaturated Fat & 0.490 \\
\hline
\end{tabular}

In the experiments, the extract by acetone can take the highest residue and mixture solvent take a high residue. The different polarities of solvent can make the difference of residue product in the extraction. The extract residue of purple corn is from extraction with three kinds of solvent such as pentane: hexane (1:1), chloroform: methanol (3:1) and acetone. 


\subsection{Determination of Flavonoids and Antioxidants by HPLC}

3.4.1 Determination the Flavonoid and Antioxidant Compounds from Chemical Extract with the Different Solvent by HPLC

Purple corn is extracted from polar and nonpolar solvent by eleven groups of solvent extraction. They are analysed and detected on flavonoids and antioxidants by HPLC. The extract is eluted by mobile phase for flavonoids (acetonitrile: 5\% formic acid: methanol: water; 75: 10: 10: 5) and antioxidants (acetonitrile: methanol; 90: 10) scaned with wavelength at $280 \mathrm{~nm}$. The different types of molecule weight by solvent separation are defined with chemical positions. These flavonoids are generally indicated by the identifying wavelength and amount absorbance of light. A qualitative property is one that defines something based on characteristics. The wavelength and amount of absorbed light can determine of flavonoid and antioxidant content. On the Table, estimate at peak area of flavonoids and antioxidants are analysed and compared with various solvent extract.

Table 4. The Peak Area (Min-Max) Flavonoid and Antioxidant by the Different Eluent

\begin{tabular}{llll}
\hline Moblie phase & $\begin{array}{l}\text { Series of Functional } \\
\text { groups }\end{array}$ & $\begin{array}{l}\text { Peak area of epicatichin } \\
\text { Min-Max (mAU) }\end{array}$ & $\begin{array}{l}\text { Total peak area } \\
\text { Min-Max (mAU) }\end{array}$ \\
\hline $\begin{array}{l}\text { ACN: } 5 \% \mathrm{HCOOH}: \mathrm{MeOH}: \\
\mathrm{H}_{2} \mathrm{O} ; 75: 10: 10: 5\end{array}$ & Flavonoids & $2.32-226.21$ & $11.34-1053.50$ \\
ACN: $\mathrm{MeOH} ; 90: 10$ & Antioxidants & $8.97-214.07$ & $27.32-1054.42$ \\
\hline
\end{tabular}

\subsubsection{Chromatogram and Chemical Fingerprints}

The pattern of the extract components of purple corn are shown some chemicals. By solvent extraction, chemical composition of different types are orderly separated by weight (\%) with HPLC. Simultaneous measurement at the same wavelength is shown that UV detector is the ability to perform spectrophotometric scanning and precise absorbance readings of wavelengths. Chemical profile or chromatogram of fingerprint is compared on the different solvent in analysis of flavonoids and antioxidants pattern. 


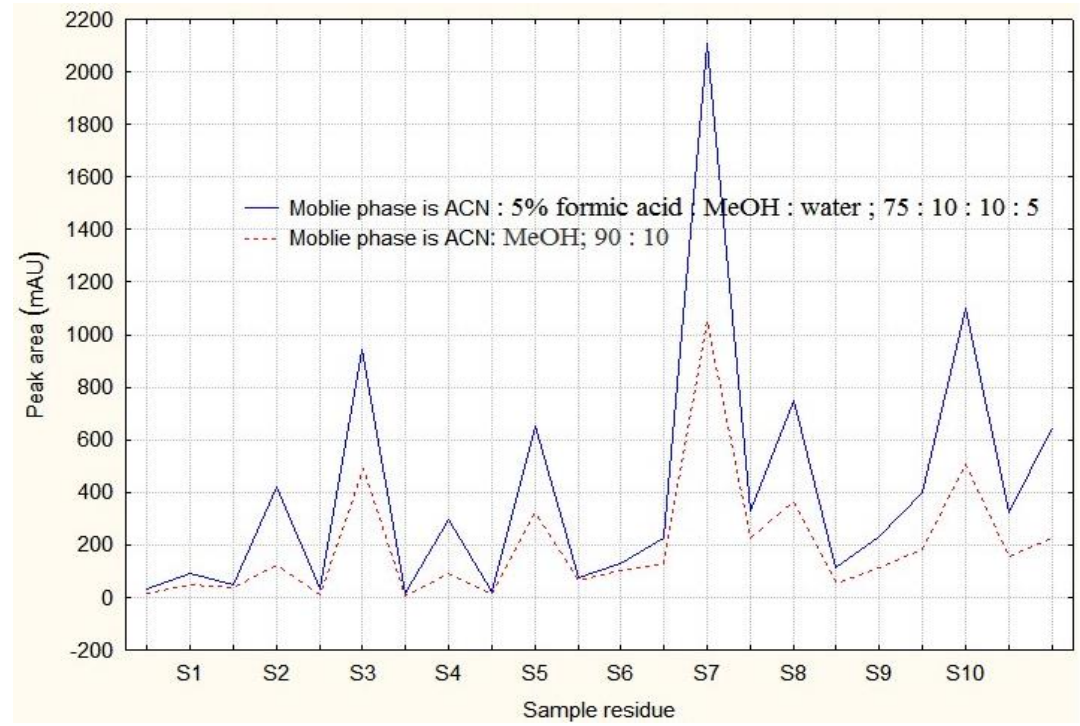

Figure 2. The Difference of Mobile Phase in Flavonoid and Antioxidant Analysis of Crude

\section{Residue}

In peak of chromatogram at $280 \mathrm{~nm}$, fingerprints allow to show height and area of peak at the difference of retention time. They are used to identify a determination for flavonoids and antioxidants. The different polarity of the extracting solvent, in each crude residue is constructed and compared on the peak area. Scanning and comparing with the profile of reference, the pattern profile is from the characteristics of wavelength by mobile phase. It is indicated some chemical components of flavonoids and antioxidants in purple corn.

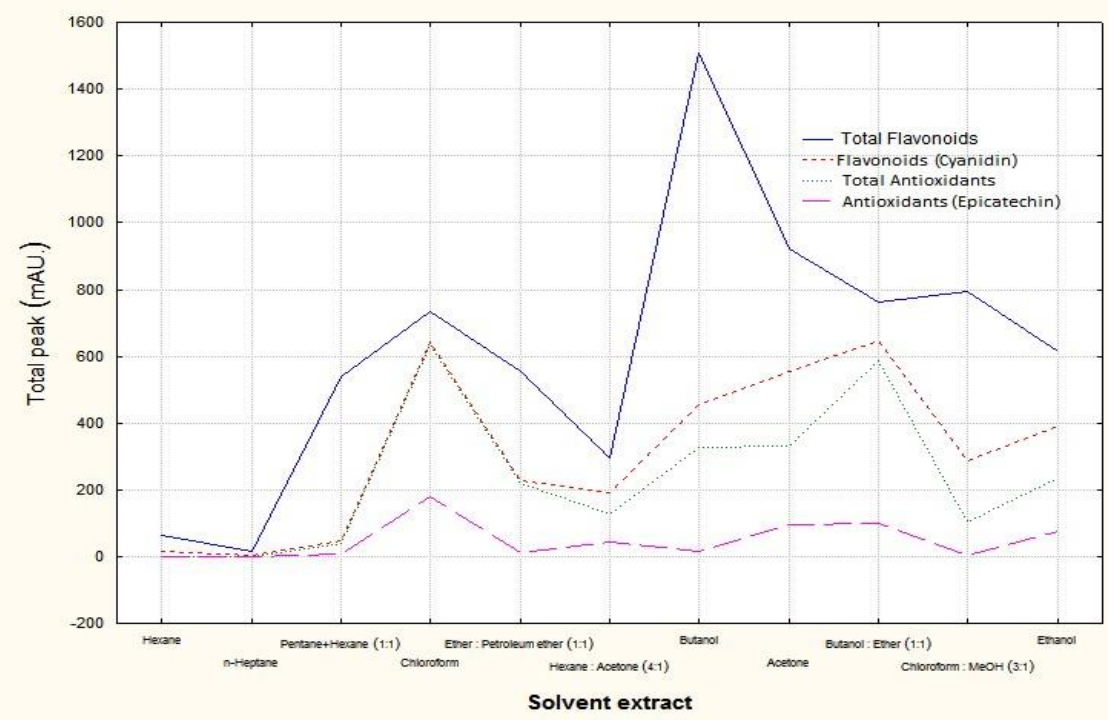

Figure 3. The Comparison on Flavonoid and Antioxidant Content by Extraction with the Difference of Solvent Extract 
On the statistic analysis, Figure 3 shows the comparison on the difference of mobile phase in flavonoid analysis. The relationships of peak area base on the different solvent of the extract. They have pearson correlation by 2 -tailed level at 0.940 and have significantly a difference by statistic at the 0.01 .

\section{Conclusion}

Purple corn has a rich composition of nutraceuticals and phytochemicals. All substances have properties as functionals that we can use to identify them. By the nature, substance characteristics base on properties of quality and unique identity. Improving and development on the changes are useful in helping to the best of raw material and products.

\section{References}

Alan, D., Mc., \& Andrew, W. (1997). Flavonoids: Isoflavonoids and neoflavonoids. IUPAC Compendium of chemical terminology (2nd ed.). Oxford: Blackwell Scientific.

AOAC. (2000). Official Methods of Analysis. The Association of Official Analytical Chemists, Arlington Virginia.

Lee, J., Rennaker, C., \& Wrolstad, R. E. (2008). Correlation of two anthocyanin quantification method: HPLC and spectrophotometric methods. Food Chemistry, 110, 782-786. https://doi.org/10.1016/j.foodchem.2008.03.010

Puminat, W., \& Teangpook, C. (2014). The acidity enhancement in extraction of anthocyanin and color of rambutan peel. The journal of food technology, 106, 220-226. 